\title{
448 - Euthanasia and Dementia
}

Beatriz Jorge; Catarina Pedro Fernandes; Juliana Carvalho; Mariana Duarte Mangas

Background: The practice of assisted dying is increasingly being discussed in a growing number of countries and is progressively regarded as a last-resort option for those suffering from severe and irreversible diseases. Recently, euthanasia in patients with psychiatric disorders and dementia has taken a prominent place in the public debate, since little is known about the prevalence and practice of euthanasia in these particular cases. Remaining a controversial subject, this study aims to describe the evolution and characteristics in reported euthanasia cases, focusing in dementia patients and the perspective from different countries regarding the nature of suffering, the voluntariness of the request and the role of the physician in the process.

Methods: A non-systematic review was performed, searching Pubmed/MEDLINE and Google Scholar for articles using the keywords dementia, euthanasia and assisted dying. Resultant articles were crossreferenced for other relevant articles not identified in the initial search.

Results: Physicians consider less likely to perform euthanasia in patients with dementia, compared to patients with a severe and life-limiting somatic illness such as cancer. Both physicians and members of the general public acknowledge difficulties in the assessment of the voluntariness of the request and the extent of suffering of patients with advanced dementia, considering that communication is compromised. While euthanasia on the grounds of unbearable suffering caused by dementia remains a comparatively limited practice, its prevalence has risen and is related to loss of dignity or the knowledge that the lasting memory of their loved ones will be of the decomposed version of oneself.

Conclusions: It is likely that the number of euthanasia requests from patients suffering from dementia and/or accumulation of health problems related to old age will continue to grow. The question of how policy makers and care providers should respond to these requests is, therefore, highly relevant, as welll as the development of practice guidelines, if medical staff is to respond adequately to these delicate requests. 